\begin{tabular}{ll|l}
\cline { 2 - 3 } & \multicolumn{2}{l}{ Intervent Neurol 2015;4:90-95 } \\
\cline { 2 - 3 } & $\begin{array}{l}\text { DOI: 10.1159/000443220 2016 S. Karger AG, Basel } \\
\text { Published online: January 6, 2016 }\end{array}$ & $\begin{array}{l}\text { 1664-9737/16/0044-0090\$39.50/0 } \\
\text { www.karger.com/ine }\end{array}$ \\
\hline
\end{tabular}

\title{
A Concealed Intracranial Aneurysm Detected after Recanalization of an Occluded Vessel: A Case Report and Literature Review
}

\author{
Sadaharu Torikoshi Yoshinori Akiyama \\ Department of Neurosurgery, Tenri Hospital, Tenri, Japan
}

\section{Key Words}

Stroke $\cdot$ Middle cerebral artery $\cdot$ Embolism $\cdot$ Thrombectomy $\cdot$ Intracranial aneurysm

\begin{abstract}
Background: Based on the results of several randomized controlled trials, acute endovascular thrombectomy is strongly recommended for patients with acute ischemic stroke due to large artery occlusion (LAO). The incidence of an intracranial aneurysm has been reported to be approximately $5 \%$ in the general population. Therefore, the possibility of the coincidence of LAO and an intracranial aneurysm at the distal part of an occluded vessel should be considered. Summary: A 74-year-old female patient presented with the sudden onset of consciousness disturbance and left-sided weakness. Neuroimages demonstrated an acute infarction due to right middle cerebral artery occlusion. The occlusion was successfully treated, and an aneurysm was incidentally detected at the occluded artery. We reviewed the literature and identified 11 cases in 8 reports, which were similar to our case. Among the 11 cases, aneurysms ruptured during endovascular therapy in 2 cases. In the present report, we discussed the prediction of concealed aneurysms and avoidance of their rupture during endovascular intervention. Key Message: The presence of an aneurysm concealed behind an embolus should be carefully assessed on preoperative and intraoperative neuroimages. The important findings for suspecting such an aneurysm are a hyperdense nodular sign on preoperative computed tomography and unusual motion of the microwire during the endovascular intervention. Even if there is no finding indicative of an aneurysm, the catheter and thrombectomy devices should be more carefully advanced than usual, especially at the common sites of aneurysms, and the devices should be appropriately chosen.
\end{abstract}

(C) 2016 S. Karger AG, Basel 


\section{Introduction}

Recently, several randomized controlled trials, such as MR CLEN and REVASCAT, have shown the overwhelming clinical benefit of acute endovascular mechanical thrombectomy for intracranial large artery occlusion (LAO) [1], and the American Heart Association/ American Stroke Association (AHA/ASA) guidelines strongly recommend that patients with acute ischemic stroke should receive endovascular therapy if they meet several criteria [2]. Therefore, endovascular therapy is becoming increasingly meaningful for acute LAO.

The presence of an intracranial aneurysm is not rare, and its incidence has been reported to be approximately $5 \%$ in the general population $[3,4]$. However, few cases of concealed intracranial aneurysms have been reported previously. Herein, we describe our experience with a case in which a concealed middle cerebral artery (MCA) aneurysm suddenly appeared during acute endovascular treatment for an acute MCA occlusion. We review the previous literature and discuss the approaches to cope with a concealed intracranial aneurysm.

\section{Case Report}

A 74-year-old, left-handed woman with a clinical history of hypertension was admitted to our emergency department with the sudden onset of disturbance of consciousness and left-sided weakness. On arrival at our hospital 40 min after symptom onset, her consciousness score was determined to be 10 on the Glasgow Coma Scale (E3, V2, M5). She presented with conjugated deviation of the eyes to the right, global aphasia, and severe left hemiplegia. Her initial National Institute of Health Stroke Scale (NIHSS) score was 21. Plain head computed tomography (CT) indicated no abnormality; however, magnetic resonance imaging (MRI) and magnetic resonance angiography demonstrated a right corona radiata infarction due to right MCA occlusion, indicating acute ischemic stroke. Her Alberta Stroke Program Early CT (ASPECT) score on diffusion-weighted images was 10 (fig. 1a). She qualified for intravenous (IV) tissue plasminogen activator (tPA) and received $0.6 \mathrm{mg} / \mathrm{kg}$ of IV alteplase $80 \mathrm{~min}$ after symptom onset. However, her symptoms did not improve. Thus, she was immediately transferred to the operating room to undergo acute endovascular therapy. A 4-Fr short sheath was placed into the right femoral artery $95 \mathrm{~min}$ after symptom onset, and right carotid angiography through a 4-Fr diagnostic catheter showed an occluded MCA as found on MRI (fig. 1b, c). The Penumbra system (Penumbra, Inc., Alameda, Calif., USA) was prepared on the table, and the 4-Fr short sheath was exchanged for a 9-Fr long sheath, along with administration of an IV heparin bolus of 3,000 U. A 9-Fr guiding catheter was introduced into the right internal carotid artery (ICA). A test injection of contrast medium 105 min after symptom onset demonstrated spontaneous recanalization of the occluded MCA, and simultaneously revealed an incidental aneurysm at the M1-M2 junction, which was $6.7 \mathrm{~mm}$ in diameter (fig. 1d). The spontaneous recanalization without the use of thrombectomy devices might have resulted from the IV tPA administration. Follow-up angiography indicated no extravasation of contrast medium, and CT performed immediately after the intervention showed no evidence of subarachnoid hemorrhage. Her symptoms gradually improved and completely disappeared the day after the intervention. Additionally, her NIHSS score reduced to 0. After the endovascular treatment, IV administration of argatroban and oral administration of aspirin, clopidogrel, and atorvastatin were initiated. She had no history of atrial fibrillation, and atrial fibrillation was not detected during hospitalization. Thoracic echocardiography revealed no abnormality. Based on these results, the probability of a cardiogenic embolism was considered to be low. Although there was no stenotic lesion at the origin of the right ICA on cerebral angiography, severe atherosclerotic lesions were noted in the intracranial ICA. The pathogenesis of the infarction was speculated to be an artery-to-artery embolism, and neck clipping of the aneurysm was performed 27 days after symptom onset.

\section{Discussion}

Wardlaw and White [3] reported that the incidence of an unruptured intracranial aneurysm was 3.6-6\% among people aged over 30 years. Iwamoto et al. [4] used data from a population-based autopsy study performed in the Japanese community of Hisayama. These 

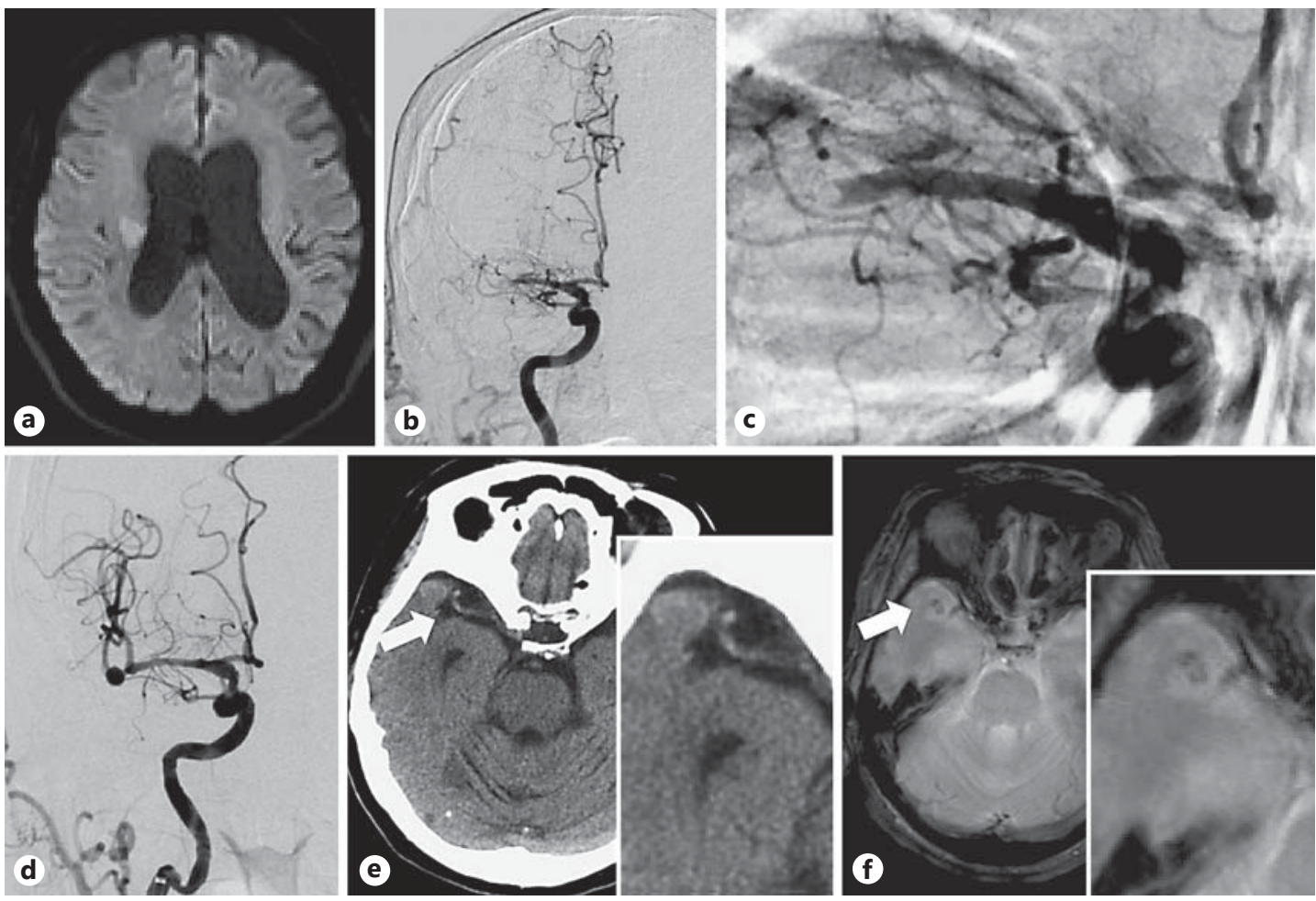

Fig. 1. a Diffusion-weighted MRI. An acute ischemic lesion is seen in the right corona radiata. b Preoperative right carotid angiography images (anteroposterior view). A filling defect is seen in the horizontal segment of the MCA. c Right carotid angiography image (anteroposterior view). Occlusion is seen at the distal end of the MCA, and the configuration of the thrombus in the occluded vessel appears as a downward convex to the distal end. d Intraoperative right carotid angiography images (anteroposterior view). Revascularization of the occluded MCA is seen. An MCA bifurcation aneurysm $6.7 \mathrm{~mm}$ in diameter is seen at the distal part of the occluded vessel. e CT image without contrast. A hyperdense nodular structure is seen at the distal end of the MCA. f T2*-weighted MRI. The structure appears to be embedded in the temporal lobe.

authors reported that the incidence of an intracranial aneurysm was $4.6 \%$, and the most frequent site was the MCA (31.5\%). Considering the incidence of unruptured aneurysms, there is a possibility that an unruptured aneurysm is concealed at the distal part of the occluded artery. We reviewed the previous literature and identified 11 cases of concealed intracranial aneurysms that were visualized after recanalization of the occluded large arteries in 8 reports, which were all case series (table 1) [5-12]. Therefore, the total number of reported cases of concealed intracranial aneurysms that appeared after recanalization of the occluded large arteries, including our case, is 12 . Among the 11 previous cases, an MCA aneurysm was detected after recanalization of an MCA occlusion in 6 cases $[5,8-10,12]$, an MCA aneurysm was detected after recanalization of an ICA occlusion in 2 cases [7], an ICA aneurysm was detected after recanalization of an ICA occlusion in 1 case [6], and a basilar artery (BA) aneurysm was detected after recanalization of a BA occlusion in 2 cases $[7,11]$. Aneurysms were visualized after acute endovascular thrombolysis in 5 cases $[6,7,9,11,12]$, after acute endovascular thrombectomy in 2 cases [5, 8], after IV tPA in 1 case [10], and after spontaneous recanalization in 3 cases $[7,8]$. Among the 11 cases, aneurysms ruptured during endovascular therapy in only 2 cases $[7,9]$. The pathogenesis was considered to be LAO in 4 cases from 3 reports $[5,6,8]$, cardiogenic embolism in 3 cases from 2 reports [6, 8], and intraaneurysmal thrombus extending distally and proximally resulting in complete parent artery 
Table 1. Previous reports on concealed intracranial aneurysms that were visualized after recanalization of the occluded large arteries

\begin{tabular}{|c|c|c|c|c|c|}
\hline Report & $\begin{array}{l}\text { Occluded } \\
\text { vessel }\end{array}$ & $\begin{array}{l}\text { Visualized } \\
\text { aneurysm }\end{array}$ & Therapy for recanalization & $\begin{array}{l}\text { Rupture } \\
\text { or not }\end{array}$ & $\begin{array}{l}\text { Neuroimaging to suspect } \\
\text { an aneurysm } \\
\text { retrospectively }\end{array}$ \\
\hline Swarnkar et al. [11] (2015) & BA & BA tip aneurysm & Thrombolysis (IA rtPA) & No & Microwire and CT \\
\hline Kuhn et al. [5] (2014) & MCA & MCA aneurysm & Thrombectomy (Penumbra) & No & CT \\
\hline Matsuzaki et al. [10] (2011) & MCA & MCA aneurysm & Thrombolysis (IV rtPA) & No & $\mathrm{CT}$ \\
\hline Ritter et al. [9] (2003) & MCA & MCA aneurysm & Thrombolysis (IA rtPA) & Rupture & CT \\
\hline Nomura et al. [12] (2002) & MCA & MCA aneurysm & Thrombolysis (urokinase) & No & Microwire \\
\hline Saito et al. [6] (2000) & ICA & ICA aneurysm & Thrombolysis (urokinase) & No & Unusual course of the ICA \\
\hline Hayashi et al. [8] (1998) & $\begin{array}{l}\text { MCA } \\
\text { MCA }\end{array}$ & $\begin{array}{l}\text { MCA aneurysm } \\
\text { MCA aneurysm }\end{array}$ & $\begin{array}{l}\text { Thrombectomy (microcatheter) } \\
\text { Spontaneous }\end{array}$ & $\begin{array}{l}\text { No } \\
\text { No }\end{array}$ & $\begin{array}{l}\text { Microwire } \\
-\end{array}$ \\
\hline Matsumaru et al. [7] (1998) & $\begin{array}{l}\text { ICA } \\
\text { ICA } \\
\text { BA }\end{array}$ & $\begin{array}{l}\text { MCA aneurysm } \\
\text { MCA aneurysm } \\
\text { BA tip aneurysm }\end{array}$ & $\begin{array}{l}\text { Thrombolysis (urokinase) } \\
\text { Spontaneous } \\
\text { Spontaneous }\end{array}$ & $\begin{array}{l}\text { Rupture } \\
\text { No } \\
\text { No }\end{array}$ & $\begin{array}{l}- \\
- \\
-\end{array}$ \\
\hline
\end{tabular}

IA = Intra-arterial; rtPA = recombinant tPA; Microwire = motion of the microwire in a U-shape during the intervention, which might be indicative of an aneurysm.

occlusion in 1 case [5]. In 4 cases from 4 reports, the authors suggested that the existence of an aneurysm could have been suspected on pretreatment CT [5, 9-11]. Our case was treated with IV tPA for acute ischemic stroke due to acute MCA occlusion and was prepared for acute endovascular thrombectomy after IV heparin bolus administration. However, recanalization of the occluded MCA was achieved without the use of the thrombectomy devices.

Kuhn et al. [5] reported that the pathogenesis of acute LAO in their case was parental artery occlusion resulting from extension of a thrombus in a concealed aneurysm. However, in our case, the configuration of the thrombus in the occluded vessel appeared as a downward convex to the distal end, as seen with cerebral angiography (fig. 1c), and therefore, we strongly suspected an acute embolism. Furthermore, Saito et al. [6] found that the lumen of an aneurysm appeared smooth after revascularization and did not show evidence of a thrombosed aneurysm. We speculated that the cause of acute LAO in our case was an artery-to-artery embolism because no evidence of a cardiogenic embolism was found and severe atherosclerotic change of the intracranial large arteries was noted with angiography and intraoperative visualization.

We might have found the right MCA aneurysm in our patient with plain CT images and T2*-weighted MRI images obtained prior to endovascular treatment. In CT images, a hyperdense nodular structure was noted continuing to the distal part of the right M1 portion consistent with the thrombus trapped in the aneurysm or an atherosclerotic change of the lumen of the aneurysm (fig. 1e), and in T2*-weighted MRI images, an altered signal intensity was noted consistent with the presence of an aneurysm embedded in the temporal lobe (fig. 1f). We believe that these features cannot be easily identified; however, we could have suspected the presence of an aneurysm if these neuroimages were reviewed in detail before the intervention. Kuhn et al. [5] showed the presence of hyperdense nodular signs consistent with a thrombus in aneurysms.

Saito et al. [6] reported that the finding of an unusual course of the ICA identified on angiography was associated with aneurysm. Additionally, Swarnkar et al. [11] suspected an 
Torikoshi and Akiyama: A Concealed Intracranial Aneurysm Detected after

Recanalization of an Occluded Vessel: A Case Report and Literature Review

invisible aneurysm during intervention owing to the unusual motion of the microwire and therefore reviewed the preoperative CT images, which revealed a hyperdense nodular sign at the tip of the BA. Hayashi et al. [8] indicated that the existence of an aneurysm might be suspected if the microwire suddenly turns back in a U-shape. Such findings indicate the possibility of a concealed aneurysm. However, in all the cases, including our case, the existence of a concealed aneurysm before initiation of endovascular therapy could not be confirmed, and therefore, there was a possibility that the concealed aneurysm would rupture during manipulation of the devices. Ritter et al. [9] reported subarachnoid hemorrhage during endovascular intervention. Moreover, 2 cases of rupture were reported previously [7, 9]. The incidence of aneurysm rupture during acute revascularization therapy, including only IV tPA, was $18 \%$ ( 2 of 11 cases). Saito et al. [6] suggested that the presence of an aneurysm should be suspected in the distal part of occluded arteries, particularly at common sites for aneurysms, such as the cisternal portion of the ICA, the bifurcation of the MCA, and the anterior communicating artery.

Cases of aneurysms at the bifurcation of the MCA were the most frequent ( 8 of 11 cases), and therefore, devices should be passed more carefully than usual through the bifurcation of the MCA during endovascular treatment for an acute MCA occlusion. Based on these previous reports, the important findings for suspecting a concealed aneurysm at the distal part of the occluded artery are a hyperdense nodular sign on preoperative CT, and an unusual motion of the microwire during the intervention. In 8 of the 12 cases, including our present case, the concealed intracranial aneurysms could have been predicted using preoperative or intraoperative neuroimages. Additionally, catheters and thrombectomy devices should be carefully passed through common sites of aneurysms.

The Penumbra system is useful for endovascular mechanical thrombectomy [13]. The reperfusion catheter of this system can be advanced to the proximal end of the thrombus to wedge the catheter with the clot without any device crossing over the lesion in order to prevent a distal embolism, and aspiration is initiated to remove the thrombus. Although the AHA/ASA guidelines strongly recommend using stent retrievers for acute LAO, the Penumbra system may be safer than stent retrievers from the point of view of preventing aneurysm rupture during endovascular treatment, as no device passes through the occluded lesion or enters into a concealed aneurismal sac. Swarnkar et al. [11] suggested that aspiration alone or in combination with thrombolysis would be more appropriate for intervention than the use of stent retrievers. The Penumbra system might be the best choice when the presence of an aneurysm can be predicted in the distal part of occluded vessels on pretreatment CT/MRI. Even if no finding indicative of an aneurysm is obtained, catheters and thrombectomy devices should be advanced carefully assuming the presence of a concealed aneurysm.

\section{Disclosure Statement}

The authors declare no conflicts of interest associated with this paper.

\section{References}

1 Ding D: Endovascular mechanical thrombectomy for acute ischemic stroke: a new standard of care. J Stroke 2015;17:123-126.

2 Powers WJ, Derdeyn CP, Biller J, Coffey CS, Hoh BL, Jauch EC, Johnston KC, Johnston SC, Khalessi AA, Kidwell CS, Meschia JF, Ovbiagele B, Yavagal DR: 2015 AHA/ASA Focused Update of the 2013 Guidelines for the Early Management of Patients with Acute Ischemic Stroke Regarding Endovascular Treatment: A Guideline for Healthcare Professionals from the American Heart Association/American Stroke Association. Stroke 2015, E-pub ahead of print. 
3 Wardlaw JM, White PM: The detection and management of unruptured intracranial aneurysms. Brain 2000; 123:205-221.

4 Iwamoto H, Kiyohara Y, Fujishima M, Kato I, Nakayama K, Sueishi K, Tsuneyoshi M: Prevalence of intracranial saccular aneurysms in a Japanese community based on a consecutive autopsy series during a 30-year observation period. The Hisayama study. Stroke 1999;30:1390-1395.

5 Kuhn AL, Hou SY, Spilberg G, Wakhloo AK: Visualization of a small hidden intracranial aneurysm during endovascular thrombectomy for acute MCA occlusion. J Vasc Interv Neurol 2014;7:47-49.

6 Saito N, Hayashi N, Okubo T, Takai K, Miyauchi H, Sasaki T, Kirino T: Internal carotid artery aneurysm visualized during successful endovascular treatment of carotid embolism. AJNR Am J Neuroradiol 2000;21:546548.

7 Matsumaru Y, Hyodo A, Okazaki M, Nose T: A pitfall of fibrinolysis. Aneurysms found after attempted fibrinolytic therapy of occluded arteries. Interv Neuroradiol 1998;4:165-169.

8 Hayashi K, Takahashi N, Furuichi S, Yoshioka T, Shibata S: Two cases of cerebral aneurysm detected after recanalization of the middle cerebral artery. No Shinkei Geka 1998;26:1103-1107.

9 Ritter MA, Kloska S, Konrad C, Droste DW, Heindel W, Ringelstein EB: Rupture of a thrombosed intracranial aneurysm during arterial thrombolysis. J Neurol 2003;250:1255-1256.

10 Matsuzaki T, Yoshino A, Sakatani K, Katayama Y: Recanalization of middle cerebral artery and intracranial aneurysm in the same ischemic territory with intravenous administration of recombinant tissue plasminogen activator: case report. J Stroke Cerebrovasc Dis 2011;20:269-272.

11 Swarnkar A, Ramaswamy R, Padalino DJ, Deshaies EM: Beware of the aneurysm in stealth mode! Neuroradiol J 2015;28:76-79.

12 Nomura H, Horie Y, Kuwayama N, Kubo M, Yamamoto H, Endo S: Incidental aneurysm detected at the occlusion site after thrombolytic therapy. Jpn J Neurosurg 2002;11:468-470.

13 Turk AS, Frei D, Fiorella D, Mocco J, Baxter B, Siddiqui A, Spiotta A, Mokin M, Dewan M, Quarfordt S, Battenhouse H, Turner R, Chaudry I: ADAPT FAST study: a direct aspiration first pass technique for acute stroke thrombectomy. J Neurointerv Surg 2014;6:260-264. 